\title{
SUBORDINATION AND $H^{p}$ FUNCTIONS
}

\author{
RAHMAN YOUNIS
}

(Communicated by Palle E. T. Jorgensen)

\begin{abstract}
Let $\phi$ and $W$ be inner functions with $\phi(0)=W(0)=0$. It is shown that if $F$ is an exposed point of the unit ball of $H^{1}$ and

$$
F\left(W\left(e^{i t}\right)\right) / F\left(\phi\left(e^{i t}\right)\right)>0
$$

almost everywhere, then $F \circ W=F \circ \phi$. If $f=z F$ such that $F$ and $1 / F$ are in $H^{r}$ and $H^{s}$, respectively, where $1 / r+1 / s \leq 2$ and $\phi$ is a finite Blaschke product, then a necessary and sufficient condition is provided in order for $f\left(W\left(e^{i t}\right)\right) / f\left(\phi\left(e^{i t}\right)\right)$ to be positive almost everywhere.
\end{abstract}

\section{INTRODUCTION}

Let $\Delta=\{z:|z|<1\}$, and let $A$ denote the set of functions analytic in $\Delta$ with the topology of uniform convergence on compact subsets of $\Delta$. Let $B_{0}$ denote the subset of $A$ consisting of all functions $\phi$ that satisfy $|\phi(z)|<1$ $(z \in \Delta)$ and $\phi(0)=0$.

For a nonconstant function $F$ in $A$, we let $s(F)$ denote the set of all functions $g$ subordinate to $F$ in $\Delta$. That is to say, $s(F)=\left\{g: g=F \circ \phi: \phi \in B_{0}\right\}$. The closed convex hull of $s(F)$ is denoted by $H s(F)$, and the set of extreme points of $H s(F)$ is denoted by $E H s(F)$.

A function $f \in A$ is said to be in the space $H^{p} \quad(0<p<\infty)$ if

$$
\|f\|_{p}=\lim _{r \rightarrow 1}\left\{\frac{1}{2 \pi} \int_{0}^{2 \pi}\left|f\left(r e^{i t}\right)\right|^{p} d t\right\}^{1 / p}<\infty .
$$

A function $W$ is called an inner function if $|W|=1$ almost everywhere on $\partial \Delta$, and it is called a finite Blaschke product if

$$
W(z)=c \prod_{i=1}^{n} \frac{z-a_{i}}{1-\bar{a}_{i} z},
$$

where $|c|=1$ and $\left|a_{i}\right|<1, i=1,2, \ldots, n$. The order of an inner function $W$ is $n$ if $W$ is a finite Blaschke product of order $n$. Otherwise, we say the

Received by the editors May 1, 1989.

1980 Mathematics Subject Classification (1985 Revision). Primary 30D55, 30C80.

Key words and phrases. Exposed points, $H^{p}$ functions, subordination. 
order of $W$ is infinity. A function $h$ in $H^{1}$ is called an outer function if

$$
h(z)=\lambda \exp \left\{\int_{0}^{2 \pi} \frac{e^{i t}+z}{e^{i t}-z} \log \left|h\left(e^{i t}\right)\right| d t\right\}, \quad|\lambda|=1
$$

A convenient reference for $H^{p}$ spaces is [7].

For $0<p \leq \infty$, the space $L^{p}$ denotes the complex Lebesgue space with respect to normalized Lebesgue measure on $\partial \Delta$. If $P$ denotes the orthogonal projection from $L^{2}$ onto $H^{2}$ and $\phi \in L^{\infty}$, then the Toeplitz operator with symbol $\phi$ is the operator $T_{\phi}$ defined on $H^{2}$ by $T_{\phi}(f)=P(\phi f), f \in H^{2}$.

For $\phi \in L^{\infty}$, we denote by $L_{\phi}$ the functional defined on $H^{1}$ by $L_{\phi}(f)=$ $(1 / 2 \pi) \int_{0}^{2 \pi} f\left(e^{i t}\right) \phi\left(e^{i t}\right) d t$. The norm of $L_{\phi}$ is $\left\|L_{\phi}\right\|=\sup \left\{\left|L_{\phi}(f)\right|:\|f\|_{1} \leq 1\right\}$. We let $S_{\phi}=\left\{f \in H^{1}:\|f\|_{1}=1\right.$ and $\left.L_{\phi}(f)=\left\|L_{\phi}\right\|\right\}$. A function $f$ in $H^{1}$ with $\|f\|_{1}=1$ is called an exposed point of the unit ball, Ball $\left(H^{1}\right)$, of $H^{1}$ if $\{f\}=S_{\phi}$, for some $\phi \in L^{\infty}$. For more details, see $[5,10,13]$.

In [8, p. 24], it was shown that if $p>1, F \in H^{p}$ and $f \in H s(F)$, with $\|f\|_{p}=\|F\|_{p}$, then $f \in s(F)$. In [2] it was shown that for $p=1$ the implication in the previous sentence is not true in general. This led to study those functions $f$ in $H^{r} \quad(0<r<\infty)$ that satisfy the following condition: If $\phi$ and $W$ are inner functions satisfying $\phi(0)=W(0)=0$ and $f\left(\phi\left(e^{i t}\right)\right) / f\left(W\left(e^{i t}\right)\right)>0$ almost everywhere, then $f \circ \phi=f \circ W$. In case $f \in H^{1}$, this condition (Theorem 1) is equivalent to the condition $\left\{g \in H s(f):\|g\|_{1}=\|f\|_{1}\right\} \subset s(f)$.

The main results of this paper are the following:

(A) Theorem 2: Let $F$ be an exposed point of Ball $\left(H^{1}\right)$. Then

(1) If $\phi$ is an inner function, then $F \circ \phi /\|F \circ \phi\|_{1}$ is an exposed point of Ball $\left(H^{1}\right)$.

(2) If $g \in H s(F)$ with $\|g\|_{1}=\|F\|_{1}$, then $g \in s(F)$.

(B) Theorem 5: Let $f=z F$ such that $F$ and $1 / F$ are in $H^{r}$ and $H^{s}$, respectively, where $1 / r+1 / s \leq 2$. Let $W$ be an inner function and $B=c \prod_{i=1}^{n}\left(z-a_{i}\right) /\left(1-\bar{a}_{i} z\right)$ be a finite Blaschke product with $W(0)=$ $B(0)=0$. Then $f\left(W\left(e^{i t}\right)\right) / f\left(B\left(e^{i t}\right)\right)>0$ almost everywhere iff $W=$ $c \prod_{i=1}^{n}\left(z-b_{i}\right) /\left(1-\bar{b}_{i} z\right)$ and $F \circ W / F \circ B=\prod_{i=1}^{n}\left(\left(1-b_{i} z\right) /\left(1-\bar{a}_{i} z\right)\right)^{2}$.

\section{SUBORDINATION AND EXPOSED POINTS}

Suppose $F \in H^{1}$, and let $\phi$ and $W$ be inner functions with $\phi(0)=W(0)=$ 0 . It follows from the proof of [2, Theorem 2] that if $F\left(\phi\left(e^{i t}\right)\right) / F\left(W\left(e^{i t}\right)\right)>0$ almost everywhere implies $F \circ \phi=F \circ W$, then for any $g \in H s(F)\|g\|_{1}=\|F\|_{1}$ implies $g \in s(F)$.

It is natural to ask whether the converse of the above result is true. The next theorem shows that this is indeed the case. 
Theorem 1. Let $F$ be in $H^{1}$. Then the following are equivalent:

(1) $\left\{g \in H s(F):\|g\|_{1}=\|F\|_{1}\right\} \subset s(F)$;

(2) If $\phi$ and $W$ are inner functions satisfying $\phi(0)=W(0)$ and

$$
F\left(\phi\left(e^{i t}\right)\right) / F\left(W\left(e^{i t}\right)\right)>0
$$

almost everywhere, then $F \circ \phi=F \circ W$.

Proof. (2) implies (1) was shown in [2]. So assume that condition (1) holds. Let $\phi$ and $W$ be inner functions satisfying $\phi(0)=W(0)=0$ and

$$
F\left(\phi\left(e^{i t}\right)\right) / F\left(W\left(e^{i t}\right)\right)>0
$$

almost everywhere. If $F \circ \phi \neq F \circ W$, let $g=\frac{1}{2} F \circ \phi+\frac{1}{2} F \circ W$. The statement $F\left(\phi\left(e^{i t}\right)\right) / F\left(W\left(e^{i t}\right)\right)>0$ almost everywhere implies that $\|F \circ \phi+F \circ W\|_{1}=$ $\|F \circ \phi\|_{1}+\|F \circ W\|_{1}$. By Ryff's Theorem [12], we have that $\|F \circ \phi\|_{1}=\|F\|_{1}=$ $\|F \circ W\|_{1}$. Thus we get $\|g\|_{1}=\|F\|_{1}$. Now condition (1) implies that $g \in s(F)$. Let $g=F \circ b$, for some $b \in B_{0}$. Since $\|g\|_{1}=\|F\|_{1}$, again by Ryff's Theorem we find that $b$ is an inner function. Since $F \in H^{1}$, by [1], $g=F \circ b \in E H s(F)$. This contradiction completes the proof that (1) implies (2).

For some outer functions, part (2) of Theorem 1 does not hold. To see this, let $f(z)=(1+z)^{4}, \phi(z)=z$, and $W(z)=-z$, and note that $f \circ \phi / f \circ W$ is positive almost everywhere on $\partial \Delta$.

In the next theorem, we show that an important class of outer functions satisfy part (2) of Theorem 1.

Theorem 2. Let $F$ be an exposed point of Ball $\left(H^{1}\right)$. Then the following statements hold:

(1) If $\phi$ is an inner function, then $F \circ \phi /\|F \circ \phi\|_{1}$ is an exposed point of Ball $\left(H^{1}\right)$.

(2) If $g \in H s(F)$ and $\|g\|_{1}=\|F\|_{1}$, then $g \in s(F)$.

The proof of (1), requires the following results:

Lemma 1 [11, Corollary 2]. Let $g$ be an outer function in $H^{1}$. Then $g /\|g\|_{1}$ is an exposed point of Ball $\left(H^{1}\right)$ if and only if $T_{|g| / g}$ is one to one.

Lemma $2\left[4\right.$, Theorem 1]. Suppose $h \in L^{\infty}$ and $\phi$ is an inner function of order $n$. Then $T_{h \circ \phi}$ is unitarily equivalent to $\oplus_{n} T_{h}$.

Proof of Theorem 2. First, we prove (1). Since $F$ is an exposed point of Ball $\left(H^{1}\right)$, then by Lemma $1, T_{|F| / F}$ is one to one. Hence $\oplus_{n} T_{|F| / F}$ is one to one, where $n$ is the order of $\phi$. By Lemma 2, $T_{|F \circ \phi| / F \circ \phi}$ is unitarily equivalent to $\oplus_{n} T_{|F| / F}$. Consequently, $T_{|F \circ \phi| / F \circ \phi}$ is one to one. Again, using Lemma 1, we find that $F \circ \phi /\|F \circ \phi\|_{1}$ is an exposed point of Ball $\left(H^{1}\right)$, as required.

To prove (2), we let $\phi$ and $W$ be inner functions satisfying $\phi(0)=W(0)=0$ and $F\left(\phi\left(e^{i t}\right)\right) / F\left(W\left(e^{i t}\right)\right)>0$ almost everywhere. Since $W(0)=\phi(0)=0$, 
then, by Ryff's Theorem, $\|F \circ \phi\|_{1}=\|F \circ W\|_{1}=1$. Since $\arg F\left(W\left(e^{i t}\right)\right)=$ $\arg F\left(\phi\left(e^{i t}\right)\right)$ almost everywhere, we have that both $F \circ \phi$ and $F \circ W$ belong to $s_{h}$, where $h$ is $|F \circ W| / F \circ W$. By part (1), $F \circ \phi$ is an exposed point of Ball $\left(H^{1}\right)$. Hence $F \circ \phi=F \circ W$. Now Theorem 1 implies that $g \in s(F)$, as required.

It is natural for someone to ask whether any outer function $f$ in $H^{1}$ satisfying condition (2) of Theorem 2 necessarily implies that $f /\|f\|_{1}$ is an exposed point of Ball $\left(H^{1}\right)$. The following theorem shows that this is not the case.

Theorem 3. There exists an outer function $f$ such that $f /\|f\|_{1}$ is not an exposed point of Ball $\left(H^{1}\right)$ and, if $g \in H s(f)$ with $\|g\|_{1}=\|f\|_{1}$, then $g \in s(f)$.

Proof. Let $f(z)=(1+z)^{2}$. On $\partial \Delta,|f| / f=|1+z|^{2} /(1+z)^{2}=(1+\bar{z}) /(1+z)=$ $\bar{z}$. Thus by [5, p. 478], $f /\|f\|_{1}$ and $z$ both belong to $s_{|f| / f}$. This shows that $f /\|f\|_{1}$ is not an exposed point of Ball $\left(H^{1}\right)$. On the other hand, let $\phi$ and $W$ be inner functions satisfying $\phi(0)=W(0)=0$ and $f\left(\phi\left(e^{i t}\right)\right) / f\left(W\left(e^{i t}\right)\right)>0$ almost everywhere. This implies that $((1+\phi) /(1+W))^{2} \cdot|1+W|^{2} /|1+\phi|^{2}=$ $\phi \cdot \bar{W}>0$ almost everywhere. Thus $\phi=W$, and so Theorem 1 completes the proof.

Examples of exposed points of ball $\left(H^{1}\right)$.

(a) Let $F$ be in $H^{1}$ that satisfies any one of the following conditions, respectively:

(1) $\operatorname{Re} F(z)>0$ for all $z \in \Delta$.

(2) $1 / F$ is in $H^{1}$,

Then $F /\|F\|_{1}$ is an exposed point of Ball $\left(H^{1}\right)$. This follows, for example, from [13,10], respectively. For more examples, see [9].

(b) Let $S=\left\{f: f\right.$ is analytic on $\Delta, f$ is one to one, $\left.f(0)=f^{\prime}(0)-1=0\right\}$. If $f=z F \in S \cap H^{1}$, then by [6, p. 33], we have $|F(z)| \geq 1 /(1+|z|)^{2}$ for all $z \in \Delta$. Thus $1 / F$ is bounded, and so, by example (a), $F /\|F\|_{1}$ is an exposed point of Ball $\left(H^{1}\right)$.

Finally, we end this section with the following result, which contains both Theorems 3 and 4 of [2].

Theorem 4. Suppose $F$ and $1 / F$ are in $H^{r}$ and $H^{s}$, respectively, where $1 / r+$ $1 / s \leq 2$. Then the following statements hold:

(a) If $\phi$ and $W$ are in $B_{0}$ and $F\left(\phi\left(e^{i t}\right)\right) / F\left(W\left(e^{i t}\right)\right)>0$ almost everywhere, then $F \circ \phi=F \circ W$.

(b) If $r=1$ and $g \in H s(F)$, with $\|g\|_{1}=\|F\|_{1}$, then $g \in s(F)$.

Proof. Let $G(z)=F(\phi(z)) / F(W(z))$, and note that $G$ is analytic on $\Delta$, $G(0)=1$, and $G\left(e^{i t}\right)>0$ almost everywhere. By [12], $F \circ \phi$ and $1 / F \circ W$ are in $H^{r}$ and $H^{s}$, respectively. If $1 / r+1 / s=2$, then we obtain $G \in H^{1 / 2}$. 
Otherwise, let $1 / 2 r+1 / 2 s+1 / p=1$, for some $p>1$. Since $|F \circ \phi|^{1 / 2} \in L^{2 r}$, $|1 / F \circ W|^{1 / 2} \in L^{2 s}$, and $1 \in L^{p}$, then by the generalized Holder's inequality, we get $|G|^{1 / 2} \in L^{1}$. Thus $G \in H^{1 / 2}$. Since $G(0)=1$ and $G\left(e^{i t}\right)>0$ almost everywhere, we conclude $[7$, p. 95] that $G=1$. Hence $F \circ \phi=F \circ W$. This proves (a).

The proof of (b) follows from (a) and Theorem 1.

\section{Properties of certain $H^{p}$ functions}

The main result in this section is Theorem 5.

Theorem 5. Suppose $f=z F$, with $F$ and $1 / F$ in $H^{r}$ and $H^{s}$, respectively, where $1 / r+1 / s \leq 2$. Let $W$ be an inner function and

$$
B(z)=c \prod_{i=1}^{n}\left(z-a_{i}\right) /\left(1-\bar{a}_{i} z\right)
$$

be a finite Blaschke product with $W(0)=B(0)=0$. Then the following conditions are equivalent:

(a) $f\left(W\left(e^{i t}\right)\right) / f\left(B\left(e^{i t}\right)\right)>0$ almost everywhere;

(b) The inner function $W$ is of the form $W(z)=c \prod_{i=1}^{n}\left(z-b_{i}\right) /\left(1-\bar{b}_{i} z\right)$ and $F(W(z)) / F(B(z))=\prod_{i=1}^{n}\left(\left(1-\bar{b}_{i} z\right) /\left(1-\bar{a}_{i} z\right)\right)^{2}, z \in D$.

Remark. The conclusion of Theorem 5 is false if $1 / r+1 / s>2$. To see this, consider the Koebe function $f(z)=z /(1-z)^{2}=z F$. Since $f \in H^{r}$ for every $r<\frac{1}{2}$, we get $F \in H^{r}$ for every $r<\frac{1}{2}$. Note that $1 / F$ is bounded. Let $W(z)=-z$ and $B(z)=z$, and note that $f\left(W\left(e^{i t}\right)\right) / f\left(B\left(e^{i t}\right)\right)>0$ because $f$ maps the boundary of $\Delta$ onto $\left\{x: x \leq-\frac{1}{4}\right\}$. Thus condition (a) in Theorem 5 holds. On the other hand, if (b) holds, then $W$ must equal $B$, which is not the case.

Before we present a proof of Theorem 5, we mention the following interesting application.

Corollary 6. Let $f=z F \in S \cap H^{1 / 2}$, and $W$ be an inner function with $W(0)=$ 0 . If $f(W(z)) / f\left(z^{2}\right)>0$ almost everywhere, then $W=z^{2}$.

Proof. Since $f$ is in $S$, by [6, p. 33], we get $|F(z)| \geq 1 /(1+|z|)^{2}$. Thus $1 / F$ is bounded. By Theorem 5, we get $W(z)=z(z-b) /(1-\bar{b} z),|b|<1$, and $F(W(z)) / F\left(z^{2}\right)=(1-\bar{b} z)^{2}$. Thus $F\left(b^{2}\right)=F\left(b^{2}\right) / F(W(b))=1 /\left(1-|b|^{2}\right)^{2}$. If $b$ were different from zero, then by [6, p. 33], $f$ would be a rotation of the Koebe function. But such a function is not in $H^{1 / 2}$. This contradiction shows that $b=0$, and thus $W=z^{2}$.

Proof of Theorem 5. First, we show that (a) implies (b). The proof is divided into two steps.

Step 1. The function $R=f \circ W / f \circ B$ can be extended to be analytic at each point of $\partial D$. 
Let $z_{0} \in \partial D$. Since $B$ is a finite Blaschke product, there exists $\delta>0$ such that $1 / B$ is analytic on $D \cap\left\{z:\left|z-z_{0}\right|<\delta\right\}$. Let $\psi$ be a conformal map of $D$ onto $D \cap\left\{z:\left|z-z_{0}\right|<\delta\right\}$, and let $I$ be the arc of $\partial D$ that corresponds to $\partial D \cap$ $\left\{z:\left|z-z_{0}\right|<\delta\right\}$ under $\psi$. Since $F \circ W / F \circ B \in H^{1 / 2}$ by the proof of Theorem 4, then $W \circ \psi(F \circ W \circ \psi) /(F \circ B \circ \psi) \in H^{1 / 2}$ by [12]. Thus $R \circ \psi$ is in $H^{1 / 2}$, and since it is positive almost everywhere on $I$, then it can be extended analytically across I [7, pp. 95-96]. Thus $R$ can be extended to be analytic at $z_{0}$. and

Step 2. The function $W$ is of the form $W(z)=c \prod_{i=1}^{n}\left(z-b_{i}\right) /\left(1-\bar{b}_{i} z\right)$,

$$
\frac{F(W(z))}{F(B(z))}=\prod_{i=1}^{n}\left(\frac{1-\bar{b}_{i} z}{1-\bar{a}_{i} z}\right)^{2}, \quad \text { for } z \in D .
$$

Since $R$ maps $\partial D$ into positive real numbers, by the argument principle we deduce that the number of zeros and the number of poles of $R$ are the same. If $b_{1}, b_{2}, \ldots, b_{n}$ are the zeros of $R$, then these are exactly the zeros of $W$, since $F \circ W$ is an outer function [7, p. 68]. The function

$$
g(z)=R \prod_{i=1}^{n} \frac{z}{\left(z-b_{i}\right)\left(1-\bar{b}_{i} z\right)} \cdot \prod_{i=1}^{n} \frac{\left(z-a_{i}\right)\left(1-\bar{a}_{i} z\right)}{z}
$$

is positive on $\partial D$, and since $g$ is analytic on $\bar{D}$ we deduce that $g=K$, for some constant $K$. Thus

$$
\begin{aligned}
R & =K \prod_{i=1}^{n}\left(z-b_{i}\right)\left(1-\bar{b}_{i} z\right) / \prod_{i=1}^{n}\left(z-a_{i}\right)\left(1-\bar{a}_{i} z\right) \\
& =c \prod_{i=1}^{n}\left(\frac{z-b_{i}}{\left.1-\bar{b}_{i} z\right)}\right) \cdot \frac{1}{B} K \prod_{i=1}^{n}\left(\frac{1-\bar{b}_{i} z}{1-\bar{a} z}\right)^{2}
\end{aligned}
$$

Since $F \circ W / F \circ B$ is outer and $R$ is in the (meromorphic) Nevalinna class, it follows, from the uniqueness of factorization, that

$$
W(z)=c \prod_{i=1}^{n}\left(z-b_{i}\right) /\left(1-\bar{b}_{i} z\right)
$$

and

$$
F(W(z)) / F(B(z))=\prod_{i=1}^{n}\left(\left(1-\bar{b}_{i} z\right) /\left(1-\bar{a}_{i} z\right)\right)^{2},
$$

since $K=(F \circ W) /(F \circ B)(0)=1$. This completes the proof that (a) implies (b). 
Finally, we show that (b) implies (a). Let $W=c \prod_{i=1}^{n}\left(z-b_{i}\right) /\left(1-\bar{b}_{i} z\right)$ and $F \circ W / F \circ B=\prod_{i=1}^{n}\left(\left(1-\bar{b}_{i} z\right) /\left(1-\bar{a}_{i} z\right)\right)^{2}$. Then

$$
\begin{aligned}
f \circ W / f \circ B & =(W / B) \prod_{i=1}^{n}\left(\left(1-\bar{b}_{i} z\right) /\left(1-\bar{a}_{i} z\right)\right)^{2} \\
& =\prod_{i=1}^{n}\left(z-b_{i}\right)\left(1-\bar{b}_{i} z\right) /\left(z-a_{i}\right)\left(1-\bar{a}_{i} z\right) \\
& =\prod_{i=1}^{n}\left|\left(z-b_{i}\right) /\left(z-a_{i}\right)\right|^{2}
\end{aligned}
$$

which is positive on $\partial \Lambda$. This completes the proof of Theorem 5 .

Remark. Theorem 5 is designed to shed some light on the following conjecture which appeared in [2]: If $f \in S \cap H^{1}$ and $\phi$ and $W$ are inner functions satisfying $\phi(0)=W(0)=0$ and $f\left(W\left(e^{i t}\right)\right) / f\left(\phi\left(e^{i t}\right)\right)>0$ almost everywhere, then $\phi=W$. Corollary 6 answers the conjecture in case $\phi(z)=z^{2}$.

\section{General Result}

In this section we prove a result of general type.

Theorem 7. Let $f=z F \in H^{1}$ such that $1 / F$ is bounded. If $W$ and $B$ are inner functions $\left(B \neq z^{n}\right)$ and $f(W(z)) / f(B(z))>0$ almost everywhere, then $(F \circ W / F \circ B)^{1 / 2}$ is in the closure of span $\left\{T_{\bar{z}} \psi: \psi\right.$ is a divisor of $\left.z B, \psi \neq z B\right\}$. Proof. Let $h^{2}=F \circ W / F \circ B$. Because $1 / F$ is bounded, then $h \in H^{2}$. Because $(W / B) h^{2}>0$ almost everywhere, then $\bar{z} \bar{B} h^{2}=\bar{z} \bar{W} h \bar{h}$. Thus, $h \in$ $\operatorname{ker}_{\overline{z B}}$. Because $\operatorname{ker} T_{\overline{z B}}=H^{2} \theta z B H^{2}$, by Corollary 6.1 of [3] we get that $h \in$ $\overline{\operatorname{span}}\left\{T_{\bar{z}} \psi: \psi\right.$ is a divisor of $z B$ and $\left.\psi \neq z B\right\}$, as required.

\section{REFERENCES}

1. Y. Abu-Muhanna, $H^{1}$ subordination and extreme points, Proc. Amer. Math. Soc. 95 (1985), 247-251.

2. Y. Abu-Muhanna and D. J. Hallenbeck, Subordination by univalent functions, Bull. London Math. Soc. 19 (1987), 249-252.

3. P. Ahern and D. Clark, On functions orthogonal to invariant subspaces, Acta Math. 124 (1970), 191-203.

4. C. Cowen, On equivalence of Toeplitz operators, J. Operator Theory 7 (1982), 167-172.

5. K. Deleeuw and W. Rudin, Extreme points and extremum problems in $H^{1}$, Pacific J. Math 8 (1958), 467-485.

6. D. Duren, Univalent functions, Springer-Verlag, 1983.

7. J. Garnett, Bounded analytic functions, Academic Press, 1981.

8. D. J. Hallenbeck and T. H. MacGregor, Linear problems and convexity techniques in geometric function theory, Pitman, 1984. 
9. J. Inoue and T. Nakazi, Polynomials of an inner function which are exposed points in $H^{1}$, Proc. Amer. Math. Soc. 100 (1987), 454-456.

10. T. Nakazi, Exposed points and extremal problems in $H^{1}$, J. Funct. Anal. 53 (1983), 224-230.

11. _ The kernel of Toeplitz operators, J. Math. Soc. Japan 38 (1980), 607-616.

12. J. Ryff, Subordination $H^{p}$ functions, Duke Math. J. 33 (1966), 347-354.

13. R. Younis, Hankel operators and extremal problems in $H^{1}$, J. Integral Equations and Operator Theory 9 (1986), 893-904.

Department of Mathematics, Kuwait University, P. O. Box 5969, 13060 Safat, Kuwait 